\title{
Contrasting Roles for Dopamine D1 and D2 Receptor Subtypes in the Dorsomedial Striatum but Not the Nucleus Accumbens Core during Behavioral Inhibition in the Stop-Signal Task in Rats
}

\author{
Dawn M. Eagle, Jacky C. K. Wong, Michelle E. Allan, Adam C. Mar, David E. Theobald, and Trevor W. Robbins \\ Behavioral and Clinical Neuroscience Institute and Department of Experimental Psychology, University of Cambridge, Cambridge CB2 3EB, United \\ Kingdom
}

\begin{abstract}
Dopamine and dopamine-receptor function are often implicated in behavioral inhibition, and deficiencies within behavioral inhibition processes linked to attention deficit/hyperactivity disorder (ADHD), schizophrenia, obsessive-compulsive disorder, and drug addiction. In the stop-signal task, which measures the speed of the process of inhibition [stop-signal reaction time (SSRT)], psychostimulant-related improvement of SSRT in ADHD is linked with dopamine function. However, the precise nature of dopaminergic control over SSRT remains unclear.

This study examined region- and receptor-specific modulation of SSRT in the rat using direct infusions of the dopamine D1 receptor (DRD1) antagonist SCH 23390 or dopamine D2 receptor (DRD2) antagonist sulpiride into the dorsomedial striatum (DMStr) or nucleus accumbens core (NAcbC). DRD1 and DRD2 antagonists had contrasting effects on SSRT that were specific to the DMStr. SCH 23390 decreased SSRT with little effect on the go response. Conversely, sulpiride increased SSRT but also increased go-trial reaction time and reduced trial completion at the highest doses. These results suggest that DRD1 and DRD2 function within the DMStr, but not the NAcbC, may act to balance behavioral inhibition in a manner that is independent of behavioral activation.
\end{abstract}

\section{Introduction}

Abnormal behavioral inhibition, e.g., the expression of impulsive or compulsive characteristics, is a defining symptom of many psychiatric and neurodegenerative conditions, including attention deficit/hyperactivity disorder (ADHD), Parkinson's disease, schizophrenia, obsessive-compulsive disorder, pathological gambling, and addiction (Aron, 2007; Everitt et al., 2008; Barnett et al., 2010; Fineberg et al., 2010; Koob and Volkow, 2010). These deficits arise from dysregulation within frontal/basal ganglia circuitry that is often linked with dopamine dysfunction (Volkow et al., 2009; Pine et al., 2010). For example, reduced dopamine D2 receptor (DRD2) expression correlates with poor behavioral control across species (Lawrence et al., 1998; Cropley et al., 2006; Dalley et al., 2007; Hamidovic et al., 2009), and decreased striatal

Received Nov. 26, 2010; revised April 1, 2011; accepted April 8, 2011.

Author contributions: D.M.E. and T.W.R. designed research; D.M.E., J.C.W., and M.E.A. performed research; A.C.M. and D.T. contributed unpublished reagents/analytic tools; D.M.E. analyzed data; D.M.E. wrote the paper.

This study was supported by a Wellcome Trust Programme Grant (089589/z/09/z) awarded to T.W.R., B. J. Everitt, B. J. Sahakian, A. C. Roberts, and J. W. Dalley and completed within the University of Cambridge Behavioral and Clinical Neuroscience Institute, supported by a joint award from the Medical Research Council and the Wellcome Trust.

This article is freely available online through the J Neurosci Open Choice option.

Correspondence should be addressed to Dawn M. Eagle, Behavioral and Clinical Neuroscience Institute and Department of Experimental Psychology, University of Cambridge, Downing Site, Cambridge CB2 3EB, UK. E-mail: de102@cam.ac.uk.

DOI:10.1523/JNEUROSCI.6182-10.2011

Copyright $\odot 2011$ the authors $\quad 0270-6474 / 11 / 317349-08 \$ 15.00 / 0$ dopamine D2/D3 receptor availability is linked to methamphetamine (Lee et al., 2009) and cocaine addiction in humans (Volkow et al., 1993) and rats (Dalley et al., 2007).

Such behavioral-inhibition deficits can be assessed using the stop-signal task, a well characterized task with high translational coherence across species, which measures the speed of the inhibition process [stop-signal reaction time (SSRT)] (Logan and Cowan, 1984). Prepotent motor responses to a go stimulus must occasionally be stopped following a stop signal, akin to stopping oneself from pressing the car accelerator pedal further if a traffic signal turns from green to red. By moving the stop signal closer to the response, it becomes more difficult to stop. Impulsive subjects have longer SSRTs, so they are less likely to stop in time (before the response is completed) compared with less-impulsive counterparts.

The putative role of dopamine in SSRT modulation arises from the effectiveness of psychostimulants (e.g., D-amphetamine, methylphenidate) to improve SSRT in ADHD (Tannock et al., 1989; de Wit et al., 2000; Feola et al., 2000). Recently, SSRT-improving effects of D-amphetamine were linked to DRD2 gene expression (Hamidovic et al., 2009). However, the precise role of dopamine in stopping is not clear. Neither the mixed dopamine D1 receptor (DRD1)/DRD2 antagonist cis-flupenthixol, nor the dopamine reuptake inhibitor GBR-12909, influenced rat SSRT (Eagle et al., 2007; Bari et al., 2009), and L-DOPA had no effect on SSRT in children with ADHD (Overtoom et al., 2003). cis-Flupenthixol also failed to alter SSRT-improving effects of either methylphenidate or modafinil in rats (Eagle et al., 2007). 
However, DRD1 and DRD2 may subserve different, even opposing, functions during SSRT modulation, similar to their roles in other forms of impulse control [e.g., in the rodent five-choice serial reaction time task (van Gaalen et al., 2006; Pattij et al., 2007; Pezze et al., 2007)]. Additionally, dopaminergic control of SSRT may be regionally specific. Excitotoxiclesion studies showed dorsomedial striatal (DMStr), but not nucleus accumbens core (NAcbC), function to be critical for SSRT in rodents (Eagle and Robbins, 2003a;b), even though the NAcbC is strongly implicated in other aspects of inhibitory control (Cardinal et al., 2001).

Here, we examined both region- and receptor-specific dopaminergic modulation of SSRT by directly infusing DRD1 or DRD2 antagonists (SCH 23390 or sulpiride) into the DMStr or NAcbC of rats. We predicted that DMStr, rather than NAcbC, dopamine function might be more critical for SSRT control.

\section{Materials and Methods \\ Subjects}

Subjects were 24 male Lister-hooded rats (Charles River), housed in groups of four in environmentally enriched cages. Experiments were conducted during the dark phase of a reversed $12 \mathrm{~h}$ light-dark cycle (lights off at 07:30). Rats weighed $260 \pm 2$ g initially ( $7-8$ weeks of age), $397 \pm 6 \mathrm{~g}$ at surgery, and $413 \pm 7 \mathrm{~g}$ at the end of the study. Weights were maintained at $\sim 95 \%$ of free-feeding weight (based on rat growth curves; Harlan). During testing, rats were fed 15-20 g of food per day (task reinforcer pellets plus laboratory chow given 1-2 $\mathrm{h}$ after the end of the daily test session), restricting weight gain to $1-2$ g per week. All experiments were conducted in accordance with the United Kingdom Animals (Scientific Procedures) Act 1986.

\section{Stop-signal task}

Rats were trained in six operant-conditioning chambers, each of which had two retractable levers, positioned to the left and right of a central food well (Med Associates). The protocol and training have been described in detail previously (Eagle and Robbins, 2003a,b). A house light in the roof of the chamber was on throughout the session. A pellet dispenser delivered $45 \mathrm{mg}$ Noyes Formula P pellets (Sandown Scientific) into the food well, and nose entry into the food well was monitored with an infrared detector. A center light, above the food well, signaled reinforcer delivery. Lights above the left and right levers signaled presentation of their respective levers. A $4500 \mathrm{~Hz}$ Sonalert tone generator (Med Associates) was mounted high on the wall opposite to the levers and food well. Control of the chambers and on-line data collection were conducted using the Whisker control system (Cardinal and Aitken, 2010) using the Stop Task program, written by A.C. Mar. Rats were tested $5 \mathrm{~d}$ per week except during drug testing (see schedule below), and performed one 25 min session per day, with a maximum of 240 trials per session.

Each trial began with a nose-poke to the central food well, after which the left lever and left light were presented (Fig. 1). A left-lever press resulted in presentation of the right lever and light and the left lever was withdrawn and the light extinguished. Rats responded rapidly between left lever and right lever presses- the go response. Response speed was maintained by limiting the time that the right lever was available - the limited hold (LH). The $\mathrm{LH}$ was set during training at a value that main- tained the maximum performance of both fast, accurate go trials and accurate no-delay stop trials. LH ranged between 1.2 and $1.4 \mathrm{~s}$ (mean \pm SEM, $1.36 \pm 0.01)$ and remained at a constant value for each rat throughout the study. During go trials, rats were rewarded with one pellet for pressing the right lever but received a timeout of $5 \mathrm{~s}$ in darkness if the right lever was not pressed within the LH period.

A stop-signal tone ( $40 \mathrm{~ms}, 4500 \mathrm{~Hz}, 80 \mathrm{~dB} \mathrm{SPL}$ ) was presented on $20 \%$ of the trials at a predetermined time between the left and right lever presses. Stop trials were randomized within the session to discourage anticipatory slowing of response speed. On stop trials, rats initiated the same response as on go trials but, following the stop signal, they were required to withhold the right lever press for the duration of the $\mathrm{LH}$ period. A correctly withheld response was rewarded with one pellet and an incorrect stop-trial response (right lever press) gave a $5 \mathrm{~s}$ timeout. On a few trials designated as stop trials, the rat responded on the right lever before the stop-signal onset (more common for late tone presentations); these trials were reclassified as go trials to maintain the overall proportion of valid stop trials in each session at $20 \%$. Rats were trained to stable baseline performance (at least 3 consecutive days of $>70 \%$ accuracy on both stop and go trials with a fixed $\mathrm{LH}$, with training completed for all rats at session 25) before the experimental protocol began. One rat failed to train effectively and was excluded from the study.

\section{Experimental design}

Before surgery, rats were tested with one set of stop-signal delays (SSDs) to generate inhibition functions and calculate SSRT. This ensured that the rats were performing the stop-signal task correctly, to conform with the constraints of the race model (Logan and Cowan, 1984). Rats first completed three no-delay sessions to calculate mean reaction time on go trials (GoRT) for each individual. SSDs for each rat were relative to its own mean GoRT, which controlled for individual differences in GoRT. 
The inhibition function was measured over five sessions with SSDs presented in pseudorandomized order (from the set GoRT-600 ms, GoRT$500 \mathrm{~ms}$, GoRT-400 ms, GoRT-300 ms, GoRT-200 ms), with one SSD per session. Rats received bilateral cannulation surgery, recovered for $5 \mathrm{~d}$ postsurgery, then were retrained to a stable baseline level of performance (7 d) and retested with one experimental set of SSDs to produce an inhibition function and calculate SSRT.

On drug testing days, rats received a two-stage session. Stage 1 was a 10 min, 80-trial session with no delay to the onset of the stop-signal in stopsignal trials, from which mean GoRT for each rat was calculated. Stage 2 began $2-3$ min after the end of stage 1 (to allow calculation of mean GoRT from stage 1 data) and SSDs were set relative to mean GoRT from stage 1, at GoRT-300 and GoRT-500 ms. Rats received a $20 \mathrm{~min}, 160$-trial session with these two SSDs presented together (but randomly ordered) within the session.

\section{Surgery}

DRD1 $(n=12)$ and DRD2 $(n=12)$ groups were matched on presurgery task performance. Rats were anesthetized with ketamine (Ketaset, 100 mg/kg i.p.; Vet Drug) and xylazine (Rompun, $10 \mathrm{mg} / \mathrm{kg}$ i.p.; Vet Drug), and secured in a stereotaxic frame fitted with atraumatic earbars, with the incisor bar set at $-3.3 \mathrm{~mm}$ relative to the interaural line to give a flat skull position. Bilateral guide cannulae (Plastics One), which consisted of a plastic body holding two 22-gauge stainless steel cannulae $3.6 \mathrm{~mm}$ apart, were implanted at the following coordinates: AP $+1.5 \mathrm{~mm}$ from bregma, lateral $\pm 1.8 \mathrm{~mm}$ from the midline, $\mathrm{DV}-1.8 \mathrm{~mm}$ from dura, calculated from a stereotaxic atlas (Paxinos and Watson, 1986). The cannulae were placed such that infusions could be made to the DMStr and subsequently to the NAcbC through the same guides. Cannulae were secured to the skull with dental acrylic and stainlesssteel screws, and wire stylets (Plastics One) occluded the guides to maintain patency. Following surgery, animals recovered in their home cages for $5 \mathrm{~d}$ before returning to testing. One rat did not recover from surgical anesthesia.

\section{Microinfusion procedure}

Stable performance was reestablished on the stop-signal task (5d). Rats then received intracerebral infusions, as described below, of either DRD1 antagonist SCH 23390 or DRD2 antagonist sulpiride (Research Biochemicals). Drugs were freshly prepared on each test day. SCH 23390 was dissolved in $0.9 \% \mathrm{NaCl}$ vehicle. Sulpiride was dissolved in acidified $0.9 \%$ $\mathrm{NaCl}$ and the final $\mathrm{pH}$ was adjusted to $\sim 7.0$ using $0.1 \mathrm{M} \mathrm{NaOH}$.

The infusion experiments were run on a weekly cycle of baseline, drug, day off, baseline, drug, day off, day off. On days off, rats received no behavioral testing and remained in their home cages. On drug testing days, for both experiments 1 and 2, rats received two phases of habituation to the cannulae before drug testing to ensure that behavioral effects relating to cannula insertion or presentation of vehicle alone were minimized. Behavioral testing began $5 \mathrm{~min}$ after the end of the drug-infusion procedure, following the methodology presented by Pezze et al. (2007).

Microinfusions were delivered through a 28-gauge bilateral injector (Plastics One) that was inserted through the guide cannula and extended $3.0 \mathrm{~mm}$ (for DMStr) or $5.0 \mathrm{~mm}$ (for NAcbC) beyond the tip of the guide cannulae. The precise infusion procedures at each stage of the experiment are described below:

\section{Experiment 1: DMStr}

Habituation ( $1 d$ ). The injector was inserted through the guide cannulae and left in place for $1 \mathrm{~min}$.

Vehicle stabilization $(1 \mathrm{~d})$. The injector was inserted and left in place for $1 \mathrm{~min}$. Rats received a $1 \mathrm{~min}(0.5 \mu \mathrm{l})$ infusion of vehicle per brain hemisphere and the injector was left in place for a further $1 \mathrm{~min}$ after infusion to allow sufficient time for diffusion into the surrounding tissue to minimize backflow along the cannula track. Rats achieved a high level of task performance ( $>200 / 240$ trials) after $1 \mathrm{~d}$ at this stage.

Drug testing phase $(4 d)$. The procedure was identical to the vehicle stabilization phase, but DRD1-group rats were infused with SCH 23390 [vehicle, 1.0, $10.0 \mathrm{ng}$ (additional dose $100 \mathrm{ng}$ ) in $0.5 \mu \mathrm{l}$ per hemisphere] and DRD2-group rats were infused with sulpiride [vehicle, 0.1, $1.0 \mathrm{ng}$ (additional dose $0.01 \mathrm{ng}$ ) in $0.5 \mu \mathrm{l}$ per hemisphere]. The first three doses of drug/vehicle were presented according to a Latin square design. Doses and infusion timings were based on a previous study (Pezze et al., 2007), with the minimum doses of SCH 23390 and sulpiride being above levels that show significant binding to their respective receptor subtypes [0.003 ng of SCH 23390 has $\sim 12.3 \%$ DRD1 occupancy and $0.005 \mathrm{ng}$ of sulpiride has $\sim 22 \%$ DRD2 occupancy (Hemsley and Crocker, 2001)]. This, and similar, methodology produced significant behavioral effects following infusions of DRD1 and DRD2 antagonists in the NAcbC (Pattij et al., 2007; Pezze et al., 2007). The final dose was determined on the basis of preliminary analysis of the other doses to select a dose that might have the maximum effect on SSRT with the minimum effect on go-trial parameters (for SCH 23390, a higher dose; for sulpiride, a lower dose).

\section{Experiment 2: NAcbC}

Vehicle stabilization $(1-2 d)$. This procedure was identical to experiment 1. Some rats failed to complete sufficient trials to permit SSRT calculation after $2 \mathrm{~d}$ of vehicle stabilization in the NAcbC site and did not continue to the drug testing phase of this experiment.

Drug testing phase ( $2 d$ ). The DRD1-group rats were infused with $\mathrm{SCH}$ 23390 (vehicle, $100 \mathrm{ng}$ in $0.5 \mu \mathrm{l}$ per hemisphere) and DRD2-group rats were infused with sulpiride (vehicle, $0.01 \mathrm{ng}$ in $0.5 \mu \mathrm{l}$ per hemisphere). Doses were selected from the set used for DMStr infusions on the basis of having a large effect on SSRT while having as small an effect as possible on go-trial parameters. Vehicle/drug presentation was counterbalanced for order-of-presentation effects.

\section{Behavioral measures}

SSRT was the main measure of behavioral inhibition (calculated from trials in which there was a delay between the start of the trial and presentation of the stop signal: a measure of the time required to inhibit the response). This was contrasted with stopping on trials in which there was no delay to the stop signal (stop-trial accuracy-more representative of a no-go-like ability to stop). In addition, we measured GoRT and the number of trials completed within a session, both in the no-delay phase (stage 1) and in the delay phase (stage 2). Drug effects on GoRT are presented for stage 2 go-trials to be directly comparable to SSRT (GoRT from stage 1 was used only to calculate SSDs for stage 2 stopsignal trials).

Although it is possible to investigate performance monitoring using the stop-signal task, our study was not designed to collect sufficient data about the relevant performance measures (i.e., posterror) for this type of analysis to be practical. The data sample size from a single session at each drug dose was too small to properly analyze performance monitoring (in terms of reaction-time distributions of posterror trial performance) without introducing significant levels of error into the analysis.

\section{Statistical analysis}

Behavioral data were subjected to ANOVA using a general linear model with significance at $\alpha=0.05$ using full-factorial models. Homogeneity of variance was verified using Levene's test. For repeated-measures analyses, Mauchly's test of sphericity was applied and the degrees of freedom corrected to more conservative values using the Huynh-Feldt epsilon for any terms involving factors in which the sphericity assumption was violated. Corrected degrees of freedom are shown to the nearest integer. Following repeated-measures analyses, simple one-way ANOVA or paired $t$ tests were used to investigate within-subjects and between-subjects factors, with $\alpha$ adjusted using Sidak's method (Howell, 1997). Spearman's ranked correlation analysis was performed to detect any interdependency of SSRT and GoRT variables. $p$ Values $>0.1$ are reported as nonsignificant (n.s.). All figures show group means with error bars of \pm 1 SEM.

\section{Assessment of cannula placement}

After behavioral testing had been completed, rats were deeply anesthetized by intraperitoneal injection of 1.5-2.0 $\mathrm{ml}$ of sodium pentobarbitone (Euthatal; May and Baker) and were transcardially perfused with 


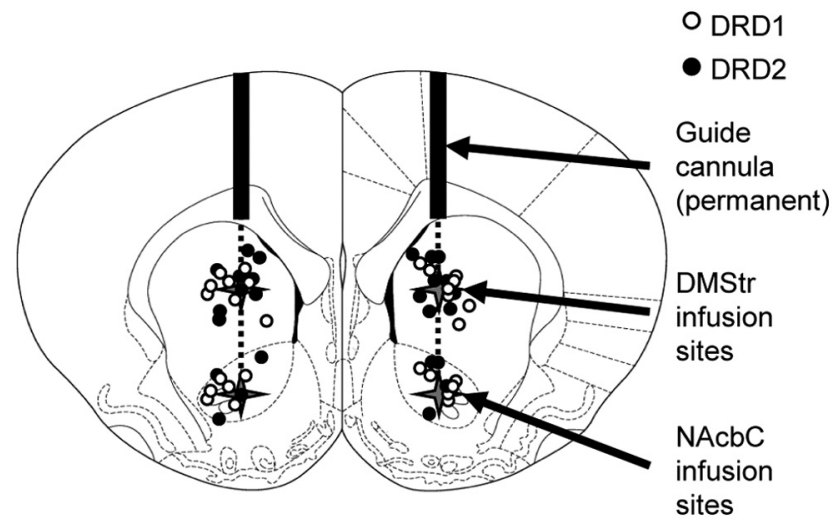

Figure 2. Schematic sections of rat brain showing cannula tip placements in the DMStr and $\mathrm{NAcb}$ for rats receiving DRD1 antagonist $\mathrm{SCH} 23390$ (white circles) or DRD2 antagonist sulpiride (black circles). Within-subject design allowed infusions to DMStr followed by NAcbC in all rats. For rats that completed both DMStr and NAcbC infusions, DMStr infusion site was estimated from the position of cannula tips in the NAcbC at the end of the experiment. Sections taken from Paxinos and Watson (1986).

$\sim 100 \mathrm{ml}$ of PBS, pH 7.4, followed by $250 \mathrm{ml}$ of formaldehyde solution [4\% (w/v) paraformaldehyde in PBS]. The brains were removed and postfixed in $4 \%(\mathrm{w} / \mathrm{v})$ formaldehyde solution for $24 \mathrm{~h}$ and then transferred to $20 \%(\mathrm{w} / \mathrm{v})$ sucrose in PBS until they sank. The tissue was serially sectioned at $60 \mu \mathrm{m}$ on a freezing-stage sledge microtome, and a 1:3 series was mounted on slides. Sections were stained with Cresyl Violet and visualized microscopically under conventional bright-field illumination. Cannula tip placements were verified for the NAcbC infusion sites and mapped onto standardized coronal sections of the rat brain (Fig. 2) (Paxinos and Watson, 1986). DMStr infusion sites were estimated from the NAcbC placements, in all rats.

\section{Exclusions and final groups}

Of 22 rats that received surgery, five were removed from experiment 1 (SCH 23390, $n=3$; sulpiride, $n=2$ ) and a further four rats were removed from experiment 2 (SCH 23390, $n=1$; sulpiride, $n=3$ ) because they failed to complete the full set of drug infusions. In experiment 1 , two rats had blockages in one or both cannulae and three rats lost the cannula head mounts in the home cages. In experiment 2 , one rat lost cannula mounts and six rats failed to perform on the task with mock or saline infusions to the NAcbC (go or stop accuracy reduced $<50 \%$ compared with noninfusion days), so it was not possible to calculate comparable SSRT values from the data. After cannula placements were assessed, one rat was removed from the DRD1 group and two rats from the DRD2 group for experiment 2 because the cannula tip extended into the nucleus accumbens shell rather than the NAcbC (these rats were included in experiment 1 as the predicted cannula placement was within the DMStr region). The final group sizes for each experiment were as follows: sulpiride: DMStr, $n=9$ and NAcbC, $n=4$; SCH 23390: DMStr, $n=8$ and NAcbC, $n=6$.

\section{Results}

The presurgical inhibition functions for the rats that completed experiment 1 (dopamine group $\times$ delay, $F_{(2,30)}=0.46$, n.s.) (Fig. $3 A$ ) and experiment 2 (dopamine group $\times$ delay, $F_{(2,14)}=0.52$, n.s.) (Fig. $3 B$ ) showed that the DRD1 and DRD2 groups were matched for inhibitory performance baseline before infusions and that there was no difference in inhibitory performance between the rats that took part in experiment 1 and those that carried on to experiment 2 [dopamine group $\times$ experiment $(1$ or 2), $F_{(1,14)}=0.69$, n.s.].

Experiment 1: infusion of dopamine receptor antagonists into the DMStr

Intra-DMStr infusions of D1 and D2 dopamine receptor antagonists had opposite effects on SSRT (Fig. 4A). The DRD1 antagonist
A

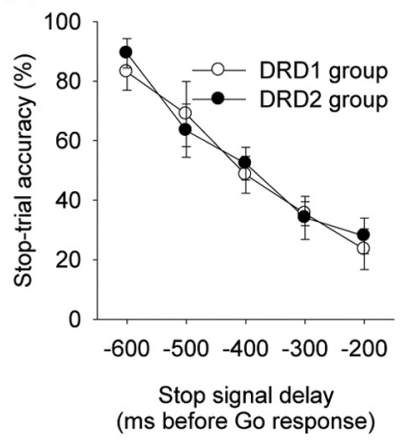

B

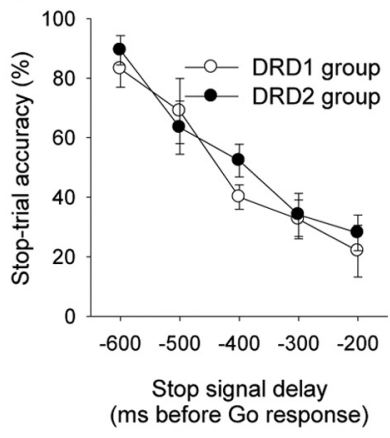

Figure 3. $\quad \boldsymbol{A}, \boldsymbol{B}$, Inhibition functions for rats that received infusions to DMStr $(\boldsymbol{A})$ and NAcbC $(\boldsymbol{B})$. There were no baseline differences in performance between the group that received DRD1 antagonist SCH 23390 and the group that received DRD2 antagonist sulpiride infusions in each phase of the experiment.

SCH 23390 decreased, or improved, SSRT (dose $F_{(3,21)}=4.43, p \leq$ $0.015 ; 0$ vs $1 \mathrm{ng}, F_{(1,7)}=9.57, p \leq 0.017,0$ vs $10 \mathrm{ng}, F_{(1,7)}=6.36$, $p \leq 0.04$; 0 vs 100 ng $F_{(1,7)}=5.17, p \leq 0.05$ ) (Fig. $4 A$ ). Conversely, the DRD2 antagonist sulpiride greatly increased, or impaired, SSRT, with the greatest effect at the highest dose (dose $F_{(3,23)}=5.20, p<0.01 ; 0$ vs $0.01 \mathrm{ng}, F_{(1,8)}=5.42, p \leq 0.05 ; 0$ vs $\left.0.1 \mathrm{ng}, F_{(1,8)}=11.53, p<0.01 ; 0 \mathrm{vs} 1 \mathrm{ng}, F_{(1,8)}=15.70, p<0.01\right)$ (Fig. 4A).

The effects of both dopamine receptor antagonists on behavioral inhibition were specific to SSRT (i.e., to the speed of the inhibitory process). Neither drug significantly affected the overall ability to inhibit, as measured by accuracy of stopping on no-delay (no-go) stop-signal trials, in which there was no delay between go-response initiation and stop-signal onset ( $\mathrm{SCH} 23390$, dose $F_{(3,21)}=2.35$, n.s.; sulpiride, dose $F_{(3,27)}=1.68$, n.s.) (Fig. $4 B$ ).

DRD1 and DRD2 antagonists had different effects on the go response, both in comparison with their respective effects on stopping and in comparison with one another. Although $\mathrm{SCH}$ 23390 affected SSRT, it had no effect on GoRT (dose $F_{(2,17)}=$ 0.04, n.s.) (Fig. 4C). In contrast, sulpiride increased GoRT, most significantly at the two highest doses (dose $F_{(3,27)}=3.36, p<$ 0.05 ; 0 vs $0.01 \mathrm{ng}, F_{(1,9)}=0.23$, n.s.; 0 vs $0.1 \mathrm{ng}, F_{(1,9)}=7.00, p \leq$ $0.03 ; 0$ vs $1 \mathrm{ng} / \mu \mathrm{l}, F_{(1,9)}=6.49, p \leq 0.03$ ) (Fig. $4 C$ ). It was unlikely that this slowing of GoRT (and slowing of SSRT) at higher doses of sulpiride was representative of a generalized motor slowing, as there was no correlation between GoRT and SSRT for any dose of sulpiride (all $r s<0.50$; all $p s>0.1$ ).

Sulpiride also significantly reduced the number of trials completed in a session; this effect was most marked during the second phase of the session (no-delay: dose $F_{(3,24)}=1.56$, n.s.; SSD: dose $F_{(3,27)}=6.25, p<0.01$ ) (Fig. $4 D$ ). Further analysis showed that this effect was maintained by the highest dose of sulpiride alone (sulpiride, 0 vs $0.01 \mathrm{ng}, F_{(1,8)}=0.01$, n.s.; 0 vs $0.1 \mathrm{ng}, F_{(1,8)}=2.48$, n.s.; 0 vs $\left.1.0 \mathrm{ng}, F_{(1,8)}=14.56, p<0.01\right)$, suggesting that the highest dose of sulpiride may have affected motivation to work for reward later in the session.

SCH 23390 had no effect on trial completion, either during the first, no-delay phase of the session $\left(\operatorname{dose} F_{(3,22)}=1.21\right.$, n.s.) or during the second, SSD phase of the session (dose $F_{(3,18)}=1.56$, n.s.).

Experiment 2: infusion of dopamine receptor antagonists into the NAcbC

When infused directly into the NAcbC, neither SCH 23390 nor sulpiride significantly affected any measure of stop-signal task 

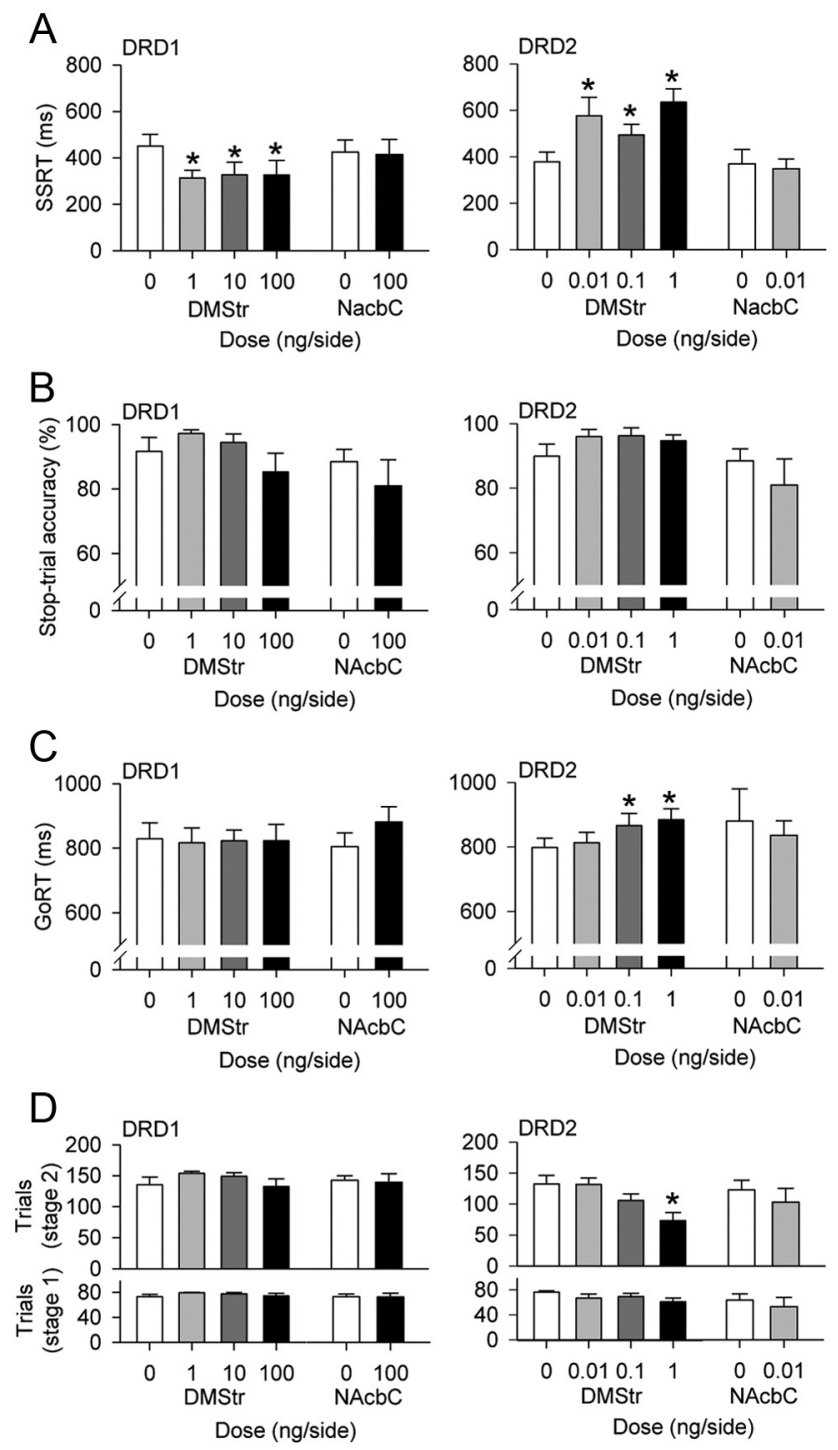

Figure 4. Behavioral effects of infusions of DRD1 antagonist SCH 23390 or DRD2 antagonist sulpiride into DMStr or NAcbC. $\boldsymbol{A}, \boldsymbol{B}$, Effects on inhibition: SSRT $(\boldsymbol{A})$ and no-go-like $(\boldsymbol{B})$ inhibition measured with no-delay to the stop signal. $\boldsymbol{C}, \boldsymbol{D}$, Effects on response activation: GoRT ( $\boldsymbol{C})$ and trial completion (D) during stage 1 (no-delay) or stage 2 (with delays) within each session. Error bars represent 1 SEM. ${ }^{*} p<0.05$ compared with vehicle.

performance. SCH 23390 had no effect on SSRT (dose $F_{(1,5)}=$ 0.09 , n.s.), GoRT (dose $F_{(1,7)}=3.07$, n.s.), or stop-trial accuracy (in no-delay, no-go trials, dose $F_{(1,7)}=1.28$, n.s.). Sulpiride had no significant effects on SSRT (dose $F_{(1,3)}=0.16$, n.s.), GoRT (dose $F_{(1,3)}=0.16$, n.s.), or no-delay stop-trial accuracy (dose $F_{(1,3)}=0.17$, n.s.). Neither drug significantly affected the total number of trials completed in a session, in the no-delay section of the session, or in the SSD section of the session (SCH 23390 no-delay dose $F_{(1,7)}=0.01$, n.s.; SCH 23390 SSD dose $F_{(1,7)}=$ 0.09 , n.s.; sulpiride no-delay dose $F_{(1,3)}=4.13$, n.s.; sulpiride SSD dose $F_{(1,3)}=4.09$, n.s.; trials slightly reduced by sulpiride in both cases).

Control for differences in group composition and baselines

Some of the rats that completed experiment 1 failed to complete experiment 2. It is possible that the DMStr-specific effects of drugs on the stop-signal task were related to this difference in subject-group composition. To examine this possible confound, we reexamined the data from experiment 1 for each drug to compare performance of the subgroup of rats that completed both experimental phases (i.e., in both DMStr and NAcbC groups) with those that were only included in the analysis for experiment 1 (i.e., only in the DMStr group). There were no significant experimental subgroup $\times$ dose effects on any behavioral measure for either SCH 23390 or sulpiride (SCH 23390: experimental subgroup $\times$ dose for SSRT, $F_{(3,18)}=0.45$, n.s.; all other measures, $F<1.68$, n.s.; sulpiride: experimental subgroup $\times$ dose for SSRT, $F_{(3,21)}=1.61$, n.s.; all other measures, $F<2.13$, n.s.). Therefore, the DMStr-specific effects of SCH 23390 and sulpiride on SSRT, GoRT, and trial completion did not result from including rats in the DMStr group that were subsequently absent from the final analysis of the NAcbC group.

Additionally, we assessed whether there were baseline differences between the two dopamine receptor antagonist groups that could account for differences in behavioral effects of each of these antagonists. There were no significant differences between DRD1 and DRD2 vehicle infusions for any measure on the stop signal task. This was the case for both experiment 1 (dopamine group SSRT, $F_{(1,15)}=1.94$, n.s.; all other measures, $F<0.55$, n.s.) and experiment 2 (dopamine group SSRT, $F_{(1,7)}=0.39$, n.s.; all other measures, $F<2.64$, n.s.).

\section{Discussion}

We showed that stopping is directly modulated by dopamine in the striatum, but that this action is dopamine receptor-specific and anatomically constrained. Dopamine D1 and D2 receptors in the dorsomedial striatum, but not nucleus accumbens core, had clearly opposing functions to control the speed with which an action was stopped (SSRT). The DRD1 antagonist SCH 23390 decreased (i.e., speeded) SSRT, allowing faster inhibition following an instruction to stop but having little or no effect on the speed or likelihood of action at other times. This suggests that, normally, dopamine acting at D1 receptors in the DMStr has a very specific function during response control: to retard the imposition of behavioral inhibition, thus making it more likely that a response is completed. Conversely, the DRD2 antagonist sulpiride increased (i.e., slowed) SSRT, suggesting that dopamine acting at D2 receptors accelerates braking of responding, making it more likely that a response is inhibited. Although the two higher doses of sulpiride also increased response time on go trials, there was no correlation between the two measures, confirming that SSRT and GoRT are independent measures and that increased SSRT is unlikely to result from generalized motor slowing.

Several recent studies have linked reduced DRD2 function with impaired behavioral inhibition and SSRT control in particular; e.g., in ADHD and chronic drug use (Volkow et al., 1993; Hamidovic et al., 2009). However, our study is the first to show the regional specificity with which DRD2 controls response braking, i.e., within the dorsomedial but not ventral striatum, the latter of which is implicated in the control of some other forms of behavioral inhibition (for review, see Eagle and Baunez, 2010). Perhaps more intriguing is that our study provides the first evidence that dopamine acting at DRD1 has an opposing action on SSRT to that of DRD2 within the same critical region of the striatum. Such a role for DRD1 is not entirely surprising, given the purported roles of DRD1 and DRD2 families of receptor subtypes in direct and indirect pathways within the basal ganglia during activation and suppression of motor behavior, respectively (Alexander and Crutcher, 1990; DeLong, 1990; Gerfen and Wilson, 1996). Dopamine facilitates striatonigral neurons through 
DRD1 and inhibits striatopallidal neurons through DRD2 (Gerfen and Wilson, 1996). Thus, according to existing models of striatal control (Frank and O'Reilly, 2006; Frank et al., 2007), dopamine might promote a cortically determined action via DRD1 in the striatum and inhibit conflicting actions via DRD2. However, we have shown an alternative mechanism for behavioral control to these existing models, which has clear anatomical specificity and may complement the model of behavioral control proposed by Frank and colleagues (2007). In a system where action and inhibition processes compete, and whichever completes first is the expressed behavior (action or inhibition, respectively) (Logan and Cowan, 1984), dopamine acting at DRD1 within the direct pathway effectively promotes action because the competing inhibition process is slowed and may not complete in time before the action occurs. Conversely, dopamine acting at DRD2 within the indirect pathway reduces action because inhibition completes more rapidly and response braking is expressed. Therefore, any change in either relative DRD1/DRD2 receptor availability or in the accessibility of dopamine to act at one or other receptor subtype would significantly influence the balance of behavioral activation/inhibition at a striatal level. Indeed, the lowest dose of sulpiride may have impaired response braking both by blocking dopamine binding at DRD2 and, via its action at presynaptic autoreceptors, by increasing dopamine availability to act at DRD1.

However, although existing models propose that that the role of striatal dopamine in inhibitory control over behavior is more prominent for the ability, or decision, to inhibit (more closely reflecting the no-go form of inhibition found in go/ no-go tasks), our study suggests that dopamine also plays a role in determining the speed at which this behavioral inhibition proceeds once the decision to stop has been made (neither DRD1 nor DRD2 blockade within the DMStr affected no-go/ no-delay inhibition in our study). Therefore, dopamine can influence inhibition both at the decision-making stage and in terms of controlling the speed of processing of the behavioral output of this decision. These subtly different models go partway toward elucidating the complex role of the striatum in behavioral control. Furthermore, as our study provides clear evidence for regional differentiation of function within the striatum, most likely reflecting differences in its cortical innervation, it is probable that information about both behavioral activation and inhibition can be integrated at different stages in the process, possibly within the striatum itself, to be subsequently output via the motor-output circuitry of the direct and indirect basal ganglia pathways.

Although the balance of DRD1/DRD2 function in the DMStr is clearly implicated in SSRT control, the NAcbC appears to play no role in the dopaminergic modulation of SSRT. This concept of a dorsal/ventral striatal division of SSRT control replicates evidence from lesion studies (Eagle and Robbins, 2003a,b), despite there being a clear role for the NAcbC in other forms of behavioral inhibition, such as impulsive-choice control and waiting (Cardinal et al., 2001; Christakou et al., 2004). For example, nearidentical methodology to this study produced marked effects of DRD1/DRD2 antagonists within the NAcbC on 5-CSRTT performance (Pezze et al., 2007), confirming that our methodology was sufficient to induce performance deficits on other measures of behavioral control. This may reflect discrete corticostriatal circuitry linked to response braking.

Further evidence from rat lesion studies shows regional differentiation within the prefrontal cortex, as well as the striatum, during SSRT control, implicating the medial division of the orbitofrontal cortex [comprising medial and ventral OFC
(m/vOFC)]. m/vOFC lesions increased SSRT, whereas lesions within the dorsomedial (prelimbic) or ventromedial (infralimbic) prefrontal cortex did not affect SSRT, despite being critical to other forms of inhibitory control (Eagle and Robbins, 2003b; Eagle et al., 2008; for review, see Eagle and Baunez, 2010; and Dalley et al., 2004). The role of the rat cingulate cortex in SSRT control is not yet known. The $\mathrm{m} / \mathrm{vOFC}$ projects strongly to DMStr but not NAcbC (Schilman et al., 2008), defining a frontostriatal circuit through which response braking may be controlled. Although the role of the OFC in behavioral inhibition is hotly debated, growing evidence points toward the $\mathrm{m} / \mathrm{vOFC}$ being functionally distinct from the more lateral division of the OFC [including lateral OFC, dorsolateral OFC, and dorsal and ventral anterior insular (AId and AIv)] (Rudebeck et al., 2008). Unlike the $\mathrm{m} / \mathrm{vOFC}$, regions within this lateral OFC subdivision project strongly to the core and shell of the nucleus accumbens (AId and AIv, respectively), rather than the DMStr (Voorn et al., 2004). This lateral OFC may have a different role from $\mathrm{m} / \mathrm{vOFC}$ in behavioral control that is unrelated to behavioral inhibition per se (for review, see Schoenbaum et al., 2009).

In human studies, ventrolateral [right inferior frontal gyrus (RIFG)] and medial prefrontal (pre-SMA/cingulate) regions are important for effective behavioral inhibition. However, the precise interplay between these structures and the anatomical circuitry underlying their integration with the basal ganglia during response braking is still not entirely clear. Several neurobehavioral and functional imaging studies proposed the hyperdirect pathway as a candidate for rapid processing of SSRT inhibition (Aron and Poldrack, 2006). This pathway links cortical regions [probably RIFG and supplementary motor area in humans, but see recent human imaging evidence against this using a functional connectivity analysis (Duann et al., 2009)] and subthalamic nucleus, each of which is implicated in SSRT control (Aron et al., 2007). It is argued that only this pathway could maintain the necessary speed of information processing to enable rapid inhibition (for review, see Chambers et al., 2009). However, our study provides the first clear evidence that temporary disruption of striatal function can severely disrupt SSRT processing speed. This adds to evidence from previous lesion studies that strongly implicates the DMStr (potentially translating to the head of the caudate in humans) in SSRT control (Eagle and Robbins, 2003a), as well as from human imaging studies that implicate the caudate during stopping (Li et al., 2008; Boehler et al., 2010). Our evidence does not oppose the possibility of hyperdirect SSRT modulation under some special circumstances. However, a hyperdirect pathway might be unnecessary for rapid processing speed, since such speeds of information transfer can be achieved perfectly well via a striatal route (Nambu et al., 2000, 2002; Chambers et al., 2009). We suggest that both direct and indirect routes through the basal ganglia may be as important as the hyperdirect pathway, or more so, for controlling the speed at which responses are inhibited, especially where additional information, such as context, can add to the appropriateness of a rapid braking process (Aron, 2010).

In summary, we have produced the first conclusive evidence that direct receptor-specific interference of dopamine transmission within the rat dorsomedial striatum affects the ability to rapidly "put the brakes on" an impending response. These findings are a significant step toward understanding how the brain controls the expression of unwanted actions and may contribute to improved treatment strategies for disorders such as ADHD and drug addiction, in which behavioral inhibition is often severely compromised. 


\section{References}

Alexander GE, Crutcher MD (1990) Functional architecture of basal ganglia circuits: neural substrates of parallel processing. Trends Neurosci 13:266-271.

Aron AR (2007) The neural basis of inhibition in cognitive control. Neuroscientist 13:214-228.

Aron AR (2010) From reactive to proactive and selective control: developing a richer model for stopping inappropriate responses. Biol Psychiatry. Advance online publication. Retrieved Oct. 8, 2010. doi:10.1016/j.biopsych.2010.07.024.

Aron AR, Poldrack RA (2006) Cortical and subcortical contributions to stop signal response inhibition: role of the subthalamic nucleus. J Neurosci 26:2424-2433.

Aron AR, Durston S, Eagle DM, Logan GD, Stinear CM, Stuphorn V (2007) Converging evidence for a fronto-basal-ganglia network for inhibitory control of action and cognition. J Neurosci 27:11860-11864.

Bari A, Eagle DM, Mar AC, Robinson ES, Robbins TW (2009) Dissociable effects of noradrenaline, dopamine, and serotonin uptake blockade on stop task performance in rats. Psychopharmacology (Berl.) 205:273-283.

Barnett JH, Robbins TW, Leeson VC, Sahakian BJ, Joyce EM, Blackwell AD (2010) Assessing cognitive function in clinical trials of schizophrenia. Neurosci Biobehav Rev 34:1161-1177.

Boehler CN, Appelbaum LG, Krebs RM, Hopf JM, Woldorff MG (2010) Pinning down response inhibition in the brain-conjunction analyses of the stop-signal task. Neuroimage 52:1621-1632.

Cardinal RN, Aitken MR (2010) Whisker: a client-server high-performance multimedia research control system. Behav Res Methods 42:1059-1071.

Cardinal RN, Pennicott DR, Sugathapala CL, Robbins TW, Everitt BJ (2001) Impulsive choice induced in rats by lesions of the nucleus accumbens core. Science 292:2499-2501.

Chambers CD, Garavan H, Bellgrove MA (2009) Insights into the neural basis of response inhibition from cognitive and clinical neuroscience. Neurosci Biobehav Rev 33:631-646.

Christakou A, Robbins TW, Everitt BJ (2004) Prefrontal cortical-ventral striatal interactions involved in affective modulation of attentional performance: implications for corticostriatal circuit function. J Neurosci 24:773-780.

Cropley VL, Fujita M, Innis RB, Nathan PJ (2006) Molecular imaging of the dopaminergic system and its association with human cognitive function. Biol Psychiatry 59:898-907.

Dalley JW, Cardinal RN, Robbins TW (2004) Prefrontal executive and cognitive functions in rodents: neural and neurochemical substrates. Neurosci Biobehav Rev 28:771-784.

Dalley JW, Fryer TD, Brichard L, Robinson ES, Theobald DE, Lääne K, Peña Y, Murphy ER, Shah Y, Probst K, Abakumova I, Aigbirhio FI, Richards HK, Hong Y, Baron JC, Everitt BJ, Robbins TW (2007) Nucleus accumbens $\mathrm{D} 2 / 3$ receptors predict trait impulsivity and cocaine reinforcement. Science 315:1267-1270.

DeLong MR (1990) Primate models of movement disorders of basal ganglia origin. Trends Neurosci 13:281-285.

de Wit H, Crean J, Richards JB (2000) Effects of D-amphetamine and ethanol on a measure of behavioral inhibition in humans. Behav Neurosci 114:830-837.

Duann JR, Ide JS, Luo X, Li CS (2009) Functional connectivity delineates distinct roles of the inferior frontal cortex and pre-supplementary motor area in stop signal inhibition. J Neurosci 29:10171-10179.

Eagle DM, Baunez C (2010) Is there an inhibitory-response-control system in the rat? Evidence from anatomical and pharmacological studies of behavioral inhibition. Neurosci Biobehav Rev 34:50-72.

Eagle DM, Robbins TW (2003a) Inhibitory control in rats performing a stop-signal reaction-time task: effects of lesions of the medial striatum and d-amphetamine. Behav Neurosci 117:1302-1317.

Eagle DM, Robbins TW (2003b) Lesions of the medial prefrontal cortex or nucleus accumbens core do not impair inhibitory control in rats performing a stop-signal reaction time task. Behav Brain Res 146:131-144.

Eagle DM, Tufft MR, Goodchild HL, Robbins TW (2007) Differential effects of modafinil and methylphenidate on stop-signal reaction time task performance in the rat, and interactions with the dopamine receptor antagonist cis-flupenthixol. Psychopharmacology (Berl.) 192:193-206.

Eagle DM, Baunez C, Hutcheson DM, Lehmann O, Shah AP, Robbins TW (2008) Stop-signal reaction time task performance: role of prefrontal cortex and subthalamic nucleus. Cereb Cortex 18:178-188.

Everitt BJ, Belin D, Economidou D, Pelloux Y, Dalley JW, Robbins TW
(2008) Neural mechanisms underlying the vulnerability to develop compulsive drug-seeking habits and addiction. Philos Trans R Soc Lond B Biol Sci 363:3125-3135.

Feola TW, de Wit H, Richards JB (2000) Effects of D-amphetamine and alcohol on a measure of behavioral inhibition in rats. Behav Neurosci 114:838-848.

Fineberg NA, Potenza MN, Chamberlain SR, Berlin HA, Menzies L, Bechara A, Sahakian BJ, Robbins TW, Bullmore ET, Hollander E (2010) Probing compulsive and impulsive behaviors, from animal models to endophenotypes: a narrative review. Neuropsychopharmacology 35:591-604.

Frank MJ, O’Reilly RC (2006) A mechanistic account of striatal dopamine function in human cognition: psychopharmacological studies with cabergoline and haloperidol. Behav Neurosci 120:497-517.

Frank MJ, Santamaria A, O'Reilly RC, Willcutt E (2007) Testing computational models of dopamine and noradrenaline dysfunction in attention deficit/hyperactivity disorder. Neuropsychopharmacology 32:1583-1599.

Gerfen, C. and Wilson, C. (1996) The basal ganglia. In: Handbook of chemical neuroanatomy, Vol 12: Integrated systems of the CNS, Part III (Swanson L, Bjorklund A, Hokfelt T, eds), pp 371-468. Amsterdam: Elsevier.

Hamidovic A, Dlugos A, Skol A, Palmer AA, de Wit H (2009) Evaluation of genetic variability in the dopamine receptor $\mathrm{D} 2$ in relation to behavioral inhibition and impulsivity/sensation seeking: an exploratory study with d-amphetamine in healthy participants. Exp Clin Psychopharmacol 17:374-383.

Hemsley KM, Crocker AD (2001) Changes in muscle tone are regulated by D1 and D2 dopamine receptors in the ventral striatum and D1 receptors in the substantia nigra. Neuropsychopharmacology 25:514-526.

Howell DC (1997) Statistical methods for psychology, 4th ed. Belmont, CA: Duxbury.

Koob GF, Volkow ND (2010) Neurocircuitry of addiction. Neuropsychopharmacology 35:217-238.

Lawrence AD, Weeks RA, Brooks DJ, Andrews TC, Watkins LH, Harding AE, Robbins TW, Sahakian BJ (1998) The relationship between striatal dopamine receptor binding and cognitive performance in Huntington's disease. Brain 121:1343-1355.

Lee B, London ED, Poldrack RA, Farahi J, Nacca A, Monterosso JR, Mumford JA, Bokarius AV, Dahlbom M, Mukherjee J, Bilder RM, Brody AL, Mandelkern MA (2009) Striatal dopamine $\mathrm{d} 2 / \mathrm{d} 3$ receptor availability is reduced in methamphetamine dependence and is linked to impulsivity. J Neurosci 29:14734-14740.

Li CS, Yan P, Sinha R, Lee TW (2008) Subcortical processes of motor response inhibition during a stop signal task. Neuroimage 41:1352-1363.

Logan GD, Cowan WB (1984) On the ability to inhibit thought and action: a theory of an act of control. Psychol Rev 91:295-327.

Nambu A, Tokuno H, Hamada I, Kita H, Imanishi M, Akazawa T, Ikeuchi Y, Hasegawa N (2000) Excitatory cortical inputs to pallidal neurons via the subthalamic nucleus in the monkey. J Neurophysiol 84:289-300.

Nambu A, Tokuno H, Takada M (2002) Functional significance of the corticosubthalamo-pallidal 'hyperdirect' pathway. Neurosci Res 43:111-117.

Overtoom CC, Verbaten MN, Kemner C, Kenemans JL, van Engeland H, Buitelaar JK, van der Molen MW, van der Gugten J, Westenberg H, Maes RA, Koelega HS (2003) Effects of methylphenidate, desipramine, and L-dopa on attention and inhibition in children with Attention Deficit Hyperactivity Disorder. Behav Brain Res 145:7-15.

Pattij T, Janssen MC, Vanderschuren LJ, Schoffelmeer AN, van Gaalen MM (2007) Involvement of dopamine D1 and D2 receptors in the nucleus accumbens core and shell in inhibitory response control. Psychopharmacology (Berl) 191:587-598.

Paxinos G, Watson C (1986) The rat brain in stereotaxic coordinates, 2nd ed. London: Academic.

Pezze MA, Dalley JW, Robbins TW (2007) Differential roles of dopamine D1 and D2 receptors in the nucleus accumbens in attentional performance on the five-choice serial reaction time task. Neuropsychopharmacology 32:273-283.

Pine A, Shiner T, Seymour B, Dolan RJ (2010) Dopamine, time, and impulsivity in humans. J Neurosci 30:8888-8896.

Rudebeck PH, Bannerman DM, Rushworth MF (2008) The contribution of distinct subregions of the ventromedial frontal cortex to emotion, social behavior, and decision making. Cogn Affect Behav Neurosci 8:485-497.

Schilman EA, Uylings HB, Galis-de Graaf Y, Joel D, Groenewegen HJ (2008) The orbital cortex in rats topographically projects to central parts of the caudate-putamen complex. Neurosci Lett 432:40-45. 
Schoenbaum G, Roesch MR, Stalnaker TA, Takahashi YK (2009 A new perspective on the role of the orbitofrontal cortex in adaptive behavior. Nat Rev Neurosci 10:885-892.

Tannock R, Schachar RJ, Carr RP, Chajczyk D, Logan GD (1989) Effects of methylphenidate on inhibitory control in hyperactive children. J Abnorm Child Psychol 17:473-491.

van Gaalen MM, Brueggeman RJ, Bronius PF, Schoffelmeer AN, Vanderschuren LJ (2006) Behavioral disinhibition requires dopamine receptor activation. Psychopharmacology (Berl.) 187:73-85.

Volkow ND, Fowler JS, Wang GJ, Hitzemann R, Logan J, Schlyer DJ, Dewey
SL, Wolf AP (1993) Decreased dopamine D2 receptor availability is associated with reduced frontal metabolism in cocaine abusers. Synapse 14:169-177.

Volkow ND, Wang GJ, Kollins SH, Wigal TL, Newcorn JH, Telang F, Fowler JS, Zhu W, Logan J, Ma Y, Pradhan K, Wong C, Swanson JM (2009) Evaluating dopamine reward pathway in ADHD: clinical implications. JAMA 302:1084-1091. Erratum: 302:1420.

Voorn P, Vanderschuren LJ, Groenewegen HJ, Robbins TW, Pennartz CM (2004) Putting a spin on the dorsal-ventral divide of the striatum. Trends Neurosci 27:468-474. 